\title{
Analysis of Metallic Nanoantennas for Solar Energy Conversion
}

\author{
B. Mora Ventura ${ }^{\mathrm{a}}$, R. Díaz de León ${ }^{\mathrm{b}}$, G. García-Torales ${ }^{\mathrm{a}}$, Jorge L. Flores ${ }^{\mathrm{a}}$, Javier Alda ${ }^{\mathrm{c}}$, and \\ Francisco Javier González . \\ ${ }^{a}$ Departamento de Electrónica, Universidad de Guadalajara, Av. Revolución No. 1500, C.P. 44430, \\ Guadalajara, Jal., México. \\ ${ }^{\mathrm{b}}$ Universidad Autónoma de San Luis Potosí, Sierra Leona, 550, Lomas 2da Sección, Building \\ CIACYT, 78210 San Luis Potosí, México. \\ ${ }^{c}$ University Complutense of Madrid, Faculty of Optics and Optometry, Applied Optics Complutense \\ Group, Avenue, Arcos de Jalón, 118, 28037 Madrid, Spain.
}

\begin{abstract}
Recently thermo-electrical nanoantennas, also known as Seebeck nanoantennas, have been proposed as an alternative for solar energy harvesting applications. In this work we present the optical and thermal analysis of metallic nanoantennas operating at infrared wavelengths, this study is performed by numerical simulations using COMSOL Multiphysics. Several different nanoantenna designs were analyzed including dipoles, bowties and square spiral antennas. Results show that metallic nanoantennas can be tuned to absorb electromagnetic energy at infrared wavelengths, and that numerical simulation can be useful in optimizing the performance of these types of nanoantennas at optical and infrared wavelengths.
\end{abstract}

Keywords: Electromagnetic radiation, thermo-electrical nanoantennas, solar energy conversion, infrared.

\section{INTRODUCTION}

Solar energy is the largest electromagnetic energy flow entering on earth. Approximately $80 \%$ of solar energy is absorbed by the earth's surface and atmosphere and it is re-emitted as infrared radiation in the $17-42 \mathrm{THz}$ spectral range, also some other artificial thermal sources such as engines, furnaces, etc., emit within the same infrared band.

The spectrum of solar radiation can be classified into three main bands: ultraviolet (UV) radiation $(f>750 \mathrm{THz})$ with less $9 \%$; visible light $(428 \mathrm{THz}<f<750 \mathrm{THz})$ where the content is approximately $39 \%$; and the remaining $52 \%$ consisting of infrared (IR) radiation $(300 \mathrm{GHz}<f<428 \mathrm{THz})^{1}$.

Over the years, several methods have been used to harvest energy from the sun. Photovoltaic (PV) cells are the most mature technology to collect and convert the solar energy into electricity ${ }^{2}$. Nevertheless, the IR wavelengths have been sub-utilized by current photovoltaic technology and this development still cannot cover the market demand on solar panels because of their low efficiency. Thus, the PV industry will need cheaper and higher efficiency technologies to meet these requirements of the solar power market. Furthermore, harvesting energy in the IR spectrum requires the use of novel techniques, which can complement current photovoltaic technology.

An alternative for solar energy harvesting is based on nanoantennas. These devices are resonant metallic structures that confine the optical energy into small volumes in an efficient way. The optical energy induces a high-frequency current on the structure ${ }^{3}$. For this reason, nanoantennas have received a significant interest as an alternative for solar energy harvesting. Additionally, nanoantennas have found many applications in the visible and infrared spectral bands due their capacity to enhance the interaction of visible light or infrared waves with nanoscale matter and confine highly localized fields ${ }^{4}$. 
Even though nanoantennas capture infrared energy in an efficient manner ${ }^{5}$ they need a mechanism, such as a rectifier to get dc power, similar to what electromagnetic receiving devices do in the microwave region ${ }^{6}$. Nanoantennas coupled to high-speed rectifiers based on tunnel barriers, also known as "rectennas", has been extensively explored during the last years as a way to convert the optical power into dc power ${ }^{7,8}$. Rectennas have inconvenient due to its low-performance, reaching efficiencies values of $10^{-9}$ and $10^{-12}$ percent for the most explored barrier'. Matching between diodes and antennas, and the diode-like behavior of the tunnel barriers are self-dependent key parameters that should be improved to increase the performance of devices ${ }^{10,11}$. By the other hand, the incorporation of nano-rectifiers to the antennas whose dimensions are around $60 \mathrm{~nm} \times 80 \mathrm{~nm}$ for rectification to $\mathrm{THz}$ frequencies requires the use of multi-level e-beam lithography processes what can conduce to not-reproducible results.

Another technique has been proposed based on the thermoelectric effect using optical nanoantennas. This technique permits to overcome the transfer energy constraint (or mismatch impedance) between the antennas and coupled fast rectifiers. It increases the efficiency of devices and reduces the technological problems arising from fabrication ${ }^{12}$. This devices are also called thermo-electrical or Seebeck nanoantennas.

Thermo-electrical nanoantennas are based by the Seebeck effect, where a current is induced all along the structure by locally increasing the temperature of the bi-metallic junction ${ }^{13}$. The heating of the junction is optically induced when the optical radiation impinges the structure, causing a non-uniform Joule heating generated by the resonant current along the nanoantennas $^{14,15}$.

In this work, we present the optical and thermal analysis of metallic nanoantennas resonating at infrared wavelengths. This study is performed by numerical simulations using COMSOL Multiphysics. We analyze three common geometries and five metals.

Section 2 describes the design and method to evaluate the thermal and optical response. Optical and thermal results are presented in section 3, and finally conclusions are given in Section 4.

\section{NUMERICAL SIMULATIONS OF NANOANTENNAS}

Numerical simulations have been used to obtain approximate solutions to problems of mathematical physics. The software that we use is based on the finite element method. This method is applicable to electromagnetic and thermal analysis.

\subsection{Design parameters of nanoantennas}

The nanoantennas designed for solar energy harvesting applications are shown in figure 1 (a), (b) and (c). These geometries are sensitive to linear polarized radiation.

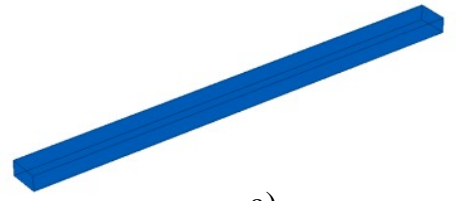

a)

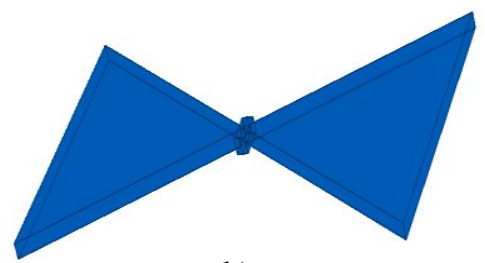

b)

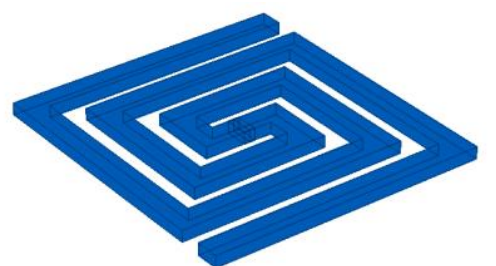

c)

Figure 1. Geometry of the proposed nanoantennas, (a) Dipole, (b) Bowtie and (c) Square spiral. 
Table 2. Parameters of the studied geometries

\begin{tabular}{|c|c|}
\hline Geometry & Parameters \\
\hline Dipole & Length $=3 \mu \mathrm{m} ;$ width $=200 \mathrm{~nm} ;$ thickness $=100 \mathrm{~nm}$ \\
\hline Bowtie & Flare angle $=60^{\circ} ;$ Length $=3 \mu \mathrm{m} ;$ width $=200 \mathrm{~nm}$; thickness $=100 \mathrm{~nm}$ \\
\hline Square spiral & $\begin{array}{c}\text { Composed by two symmetrical arms } 10.2 \mu \mathrm{m} \text { lineal long; width=200 } \mathrm{nm} ; \\
\text { thickness }=100 \mathrm{~nm}\end{array}$ \\
\hline
\end{tabular}

We study the effect of these geometries, their performance is analyzed with the induced current and electric field distribution as a function of frequency.

\subsection{Optical analysis}

The most popular materials utilized to build antennas are gold, silver and cooper. At the optical and infrared regions, metals are no longer considered to be perfect conductors and they have frequency dependent dielectric properties.

In this work, we use gold, silver, copper, titanium and nickel as the materials for designing.

The Drude model describes the optical permittivity of the metal as:

$$
\varepsilon(\omega)=\varepsilon_{\infty}-\frac{\left(\omega_{p}\right)^{2}}{\omega^{2}-i \omega \omega_{\tau}}
$$

Where $\varepsilon_{\infty}$ represents the contribution of the bound electrons to the relative dielectric constant, $\omega_{p}$ is the plasma frequency and $\omega_{\tau}$ is the damping frequency. The optical parameters are frequency-dependent as shown in (Eq. 1), however, it is previously approved in the research literature ${ }^{16}$ that the material parameters at a single frequency close to the resonance can be used instead of the frequency-dependent variable $\varepsilon(\omega)$. An acceptable agreement is maintained as long as the imaginary part of $\varepsilon$ is included. For this work we obtained the optical parameters of metals at IR frequencies previously reported in the literature ${ }^{16-19}$.

The electromagnetic simulation was performed by propagating a linearly polarized plane wave that match with the structure. The electric-field-amplitude of the plane wave was $1 \mathrm{~V} / \mathrm{m}$ and the induced current in the antenna as a function of the plane wave's frequency was measured by integrating the surface current density over the antenna.

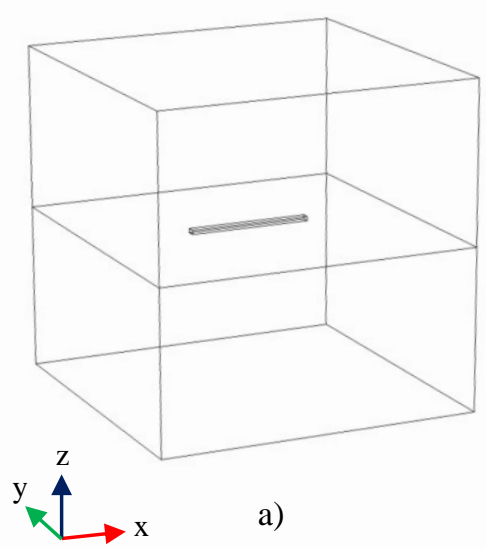

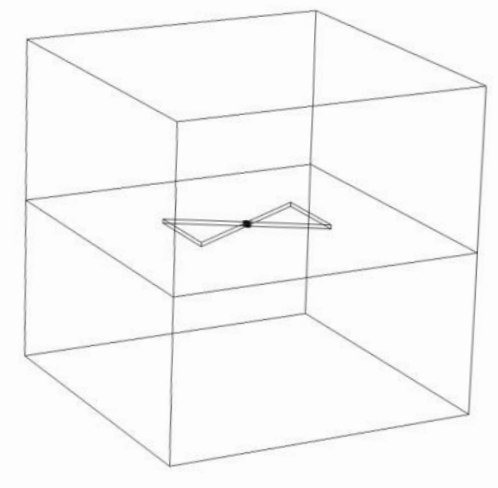

b)

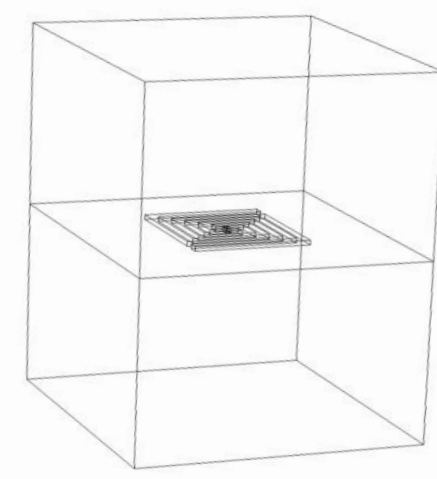

c)

Figure 2. Geometries of nanoantenas that are immersed to the physic space with electromagnetic characteristics of air, we use relative permittivity $\left(\varepsilon_{\mathrm{r}}=1\right)$, relative permeability $\left(\mu_{\mathrm{r}}=1\right)$ and electric conductivity $(\sigma=0 \mathrm{~S} / \mathrm{m})$.

The optical study lead us to analize the performance of the nanoantennas that is useful for calculate the parameters used in thermal analysis. In the next section, we present the relation between electromagnetics and thermal power. 


\subsection{Thermal analysis}

In the thermal study of the nanoantennas, we have to take into account that the incoming electromagnetic radiation into the metallic structure generates heat. The heating of the nanoantenna is described by the Joule effect. The power dissipated by the device is seen as a heat source ${ }^{20,21}$.

The total heat produced after excitation is given by the following relation:

$$
Q=\int_{v} q(x, y, z) d v=\int_{v} \sigma_{\omega}\left|\vec{E}_{\omega}(x, y, z)\right|^{2} d v,
$$

Where $\sigma(\omega)$ is the conductivity of the metal at the frequency of the incident wave, where $\vec{E}_{\omega}(x, y, z)$ is the spatial distribution of the electric field of the electromagnetic wave, and $v$ is the antenna volume.

The electric field distribution, $\vec{E}_{\omega}(x, y, z)$, is obtained after solving the following wave equation:

$$
\nabla \times\left(\frac{1}{\mu_{r}} \nabla \times \vec{E}\right)-k_{0}^{2}\left(\varepsilon_{r}+i \frac{\sigma(\omega)}{\varepsilon_{0} \omega}\right) \vec{E}=0,
$$

To find out the temperature change of the metal structure, we need to use the heat transfer equation:

$$
d C_{p} \frac{\partial T}{\partial t}=\nabla(\kappa \nabla T)+q
$$

Where $d$ is the mass density, $C_{p}$ is the heat capacity, $\kappa$ is the thermal conductivity and $q=q(x, y, z)$ is the power heat density $\left(\mathrm{W} / \mathrm{m}^{3}\right)$, which is related with the power dissipation given in Eq. $(2)^{22}$.

For the thermal analysis, we simulate the total heat produced by Joule effect with a heat source implemented in the design of the nanoantennas. The numerical model was defined using all the optical, thermal and thermo-electrical properties of materials reported at $10.6 \mu \mathrm{m}$ wavelength ${ }^{23}$.

In the thermal part of the simulation study, we used dipole nanoantennas joining their arms with two dissimilar metals. These devices are like thermocouples composed of the following materials: silver-nickel, gold-nickel, copper-nickel and titanium-nickel.

Materials with a considerable difference in their Seebeck coefficients $S_{a}$ and $S_{b}$ (property that determines the performance of materials to generate voltage from heat gradients) should be considered in order to increase the response of the devices, given by ${ }^{24,25}$.

$$
V_{o c}=\left(S_{a}-S_{b}\right) \Delta T
$$

Where $\Delta T$ is the temperature difference between the center (hot spot) and the open ends of the antenna (cold spots).

The effective temperature increase of the bi-metallic junction $\Delta T$ was found and the voltage response of the antenna was evaluated by using the Seebeck effect relationship ${ }^{25}$.

We use proprieties of nickel as a main building material, because it has a negative Seebeck coefficient and consequently it shows a considerable difference substituting the values of the materials in (Eq. 5).

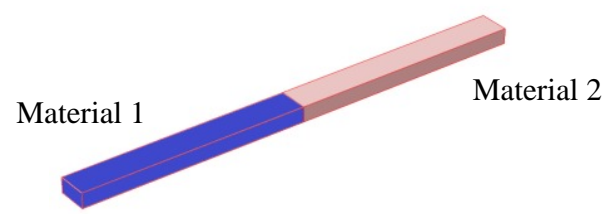

Figure 3. Schematic representation of thermoelectric dipole nanoantennas. This geometry is made of two dissimilar metals that are represented with different color, it creates a thermocouple. Each arm of the dipole nanoantenna has $200 \mathrm{~nm}$ width $\times 100 \mathrm{~nm}$ thickness and $1.5 \mu \mathrm{m}$ length. 


\section{RESULTS}
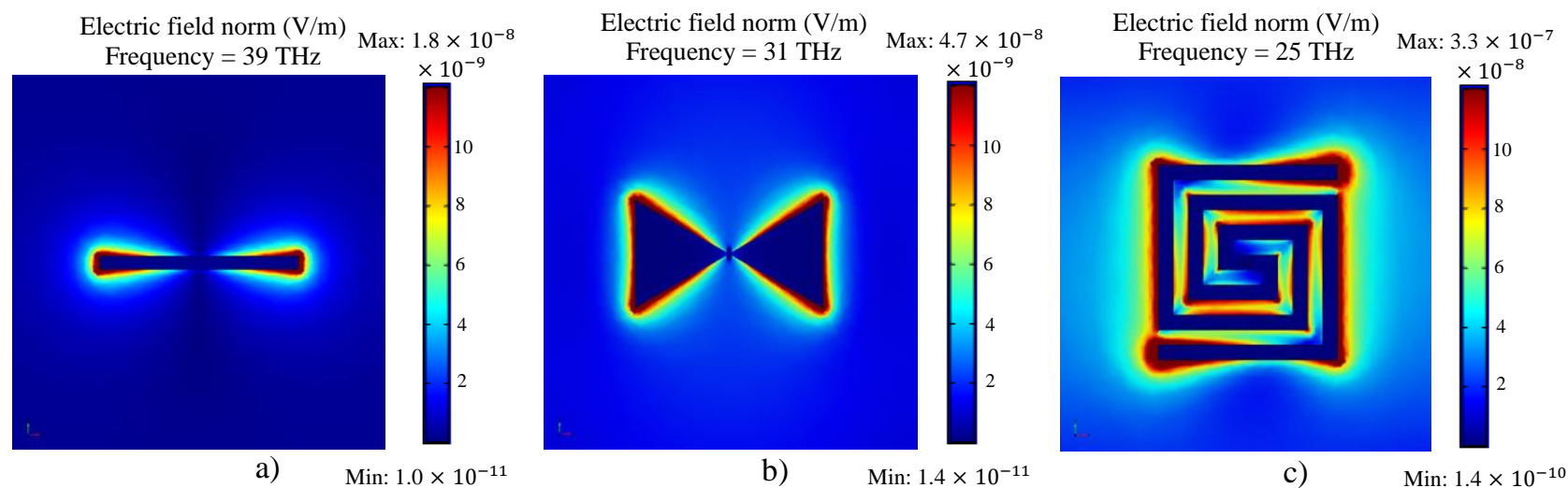

Figure 4. Electric field norm around the nanoantenna under resonant frequency condition.

Using the finite element method numerical simulation was realized, getting like result the electric field distribution for three types of nanoantennas. Addition, the induced current as a function of frequency was obtained. Figure 4 shows the electric field concentration of gold nanoantennas as a function of their resonance frequency. As expected, the highest electric field intensity is distributed along the edges of the nanostructures. The magnitude of the induced current is proportional to the electric field intensity.

It can be seen from Figure 4 that there is a resonance shift from $39 \mathrm{THz}$ for dipole, $31 \mathrm{THz}$ for bowtie and $25 \mathrm{THz}$ for square spiral antenna. These resonance frequencies correspond at infrared spectrum.

The results show in figure 5 the current induced at range of frequencies $10 \mathrm{THz}$ to $150 \mathrm{THz}$. The spiral square antenna has the largest value compared with the bowtie and the dipole nanoantennas. The higher response is due to the larger collection area in comparison with the dipole. Bowtie antenna resonates at a lower frequency than dipole antenna of the same length. Through modifying geometries is possible have different broadband of energy harvested and different resonance frequency.

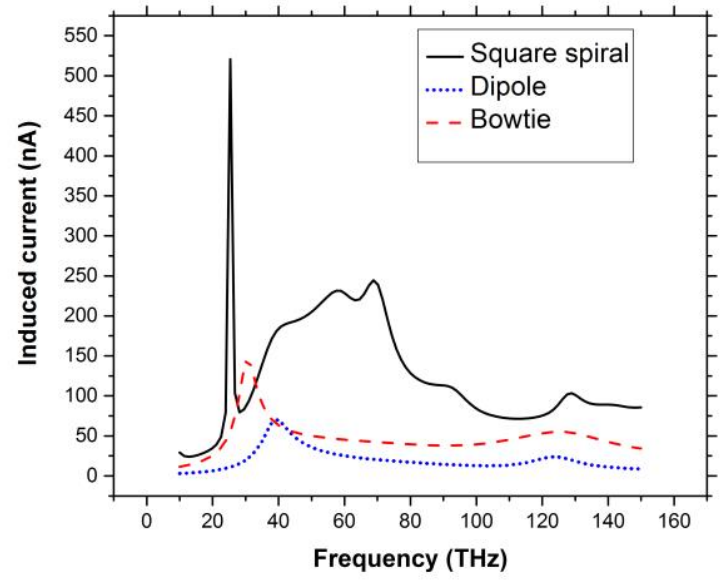

Figure 5. Induced current by a linearly polarized plane wave on gold dipole, bowtie and square spiral nanoantennas as a function of frequency. 


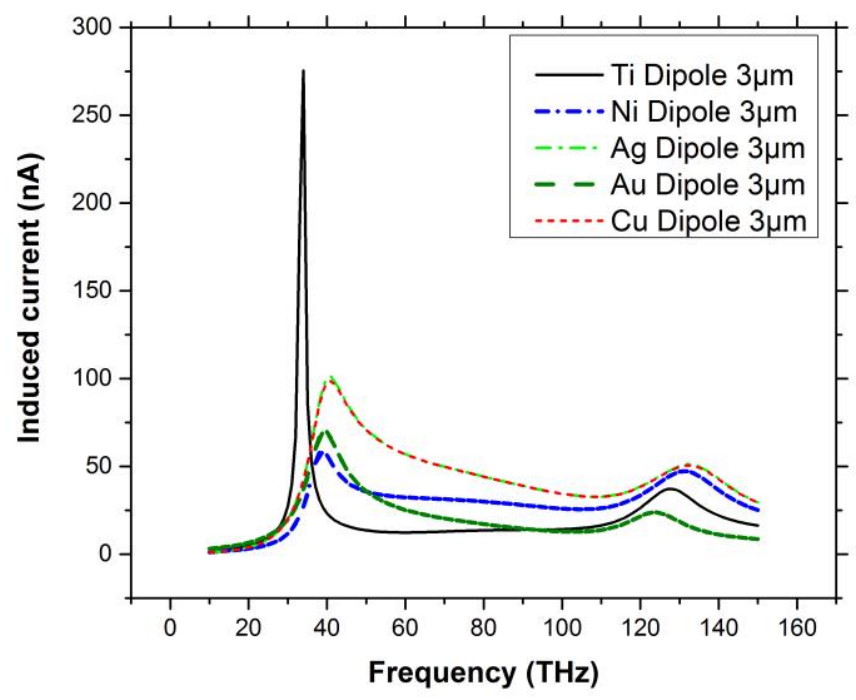

Figure 6. Induced current by a linearly polarized plane wave on gold, cooper, silver, titanium and nickel dipoles as a function of frequency.

In order to find out the influence of the material, the simulations were performed using the optical proprieties of the metal reported in the literature ${ }^{16-19}$. Figure 6 shows the effect of the material in the current induced on the nanostructures at infrared frequencies. The response of titanium, of dipoles nanoantennas with the same length, has the higher induced current. The resonance frequency is shifted in function of the type of material used.

The results of the finite-element thermal simulations are employed to evaluate the Seebeck voltage on the dipole nanoantenas. For thermal simulations the thermal conductivities of the materials were considered to work at room temperature. We obtain the temperature distribution along the dipole nanoantenna with different materials. Figure 7 shows the temperature distribution of the Ti-Ni nanoantena at the steady-state.

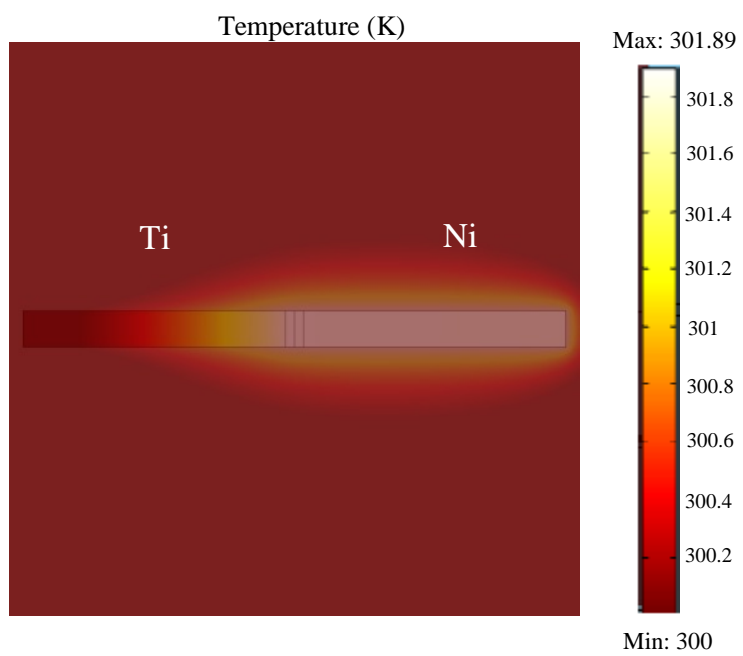

Figure 7. Thermal simulation showing the temperature distribution in dipole nanoantennas composed of a Ti-Ni bimetallic junction. 


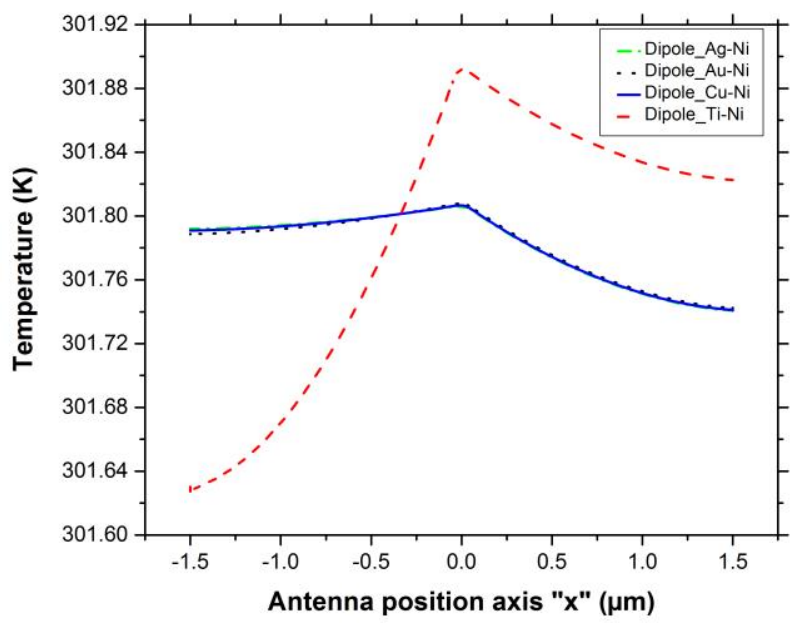

Figure 8. Temperature obtained in dipole nanoantennas of the metals analyzed in this work.

The figure 8 shows that the Ti-Ni junction has higher difference of temperature obtained of the ends of dipole nanoantenna. In figure 9, we plot the temperature for each junction. The temperature asymmetry of arms arises from the difference in thermal conductivities of used metals what causes one arm to conduct faster the heat.

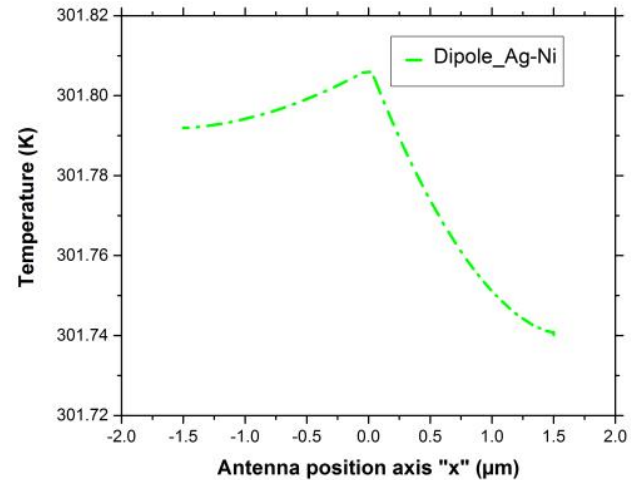

a)

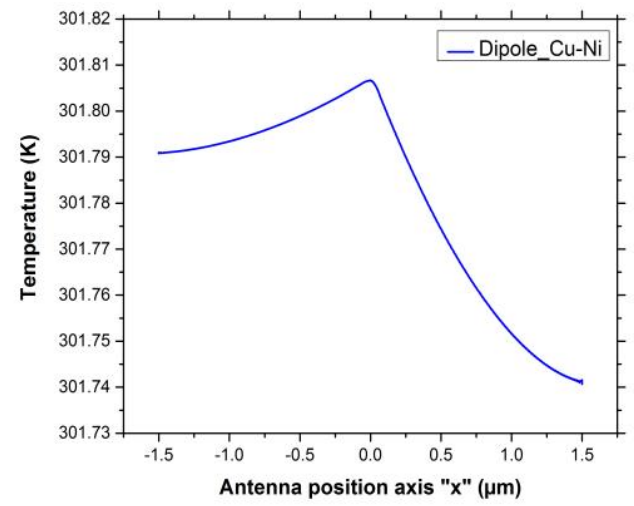

c)

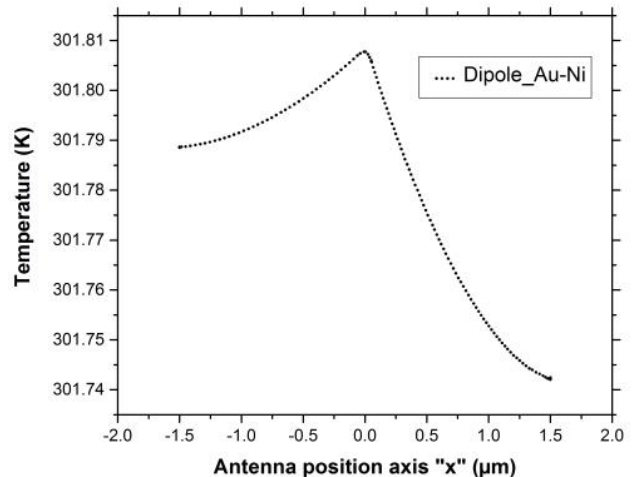

b)

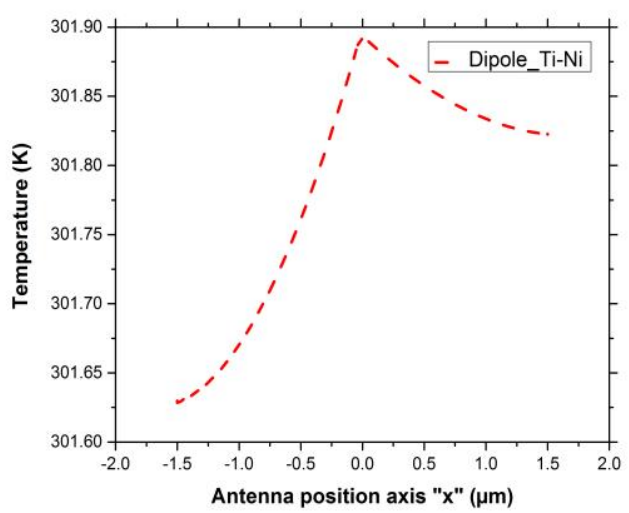

d)

Figure 9. Temperature obtained in dipole nanoantenna, (a) Silver-Nickel, (b) Gold-Nickel, (c) Copper-Nickel and (d) TitaniumNickel. 
Table 2 shows the effective temperature increase of the bi-metallic junction, and the voltage response of the antenna was evaluated by using the Seebeck effect, described in (Eq. 5). The Seebeck coefficients were taken as bulk $\left(S_{A g}=S_{A u}=\right.$ $S_{C u}=6.5 \mu \mathrm{V} / \mathrm{K}, S_{T i}=7.2 \mu \mathrm{V} / \mathrm{K}$ and $\left.S_{N i}=-15 \mu \mathrm{V} / \mathrm{K}\right)$.

Table 2. Difference temperature obtained of the ends of nanoantennas and Seebeck voltage of dipole nanoantennas with $3 \mu \mathrm{m}$ of length.

\begin{tabular}{|c|c|c|}
\hline Material junction & $\Delta \mathbf{T}(\mathbf{K})$ & $\boldsymbol{V}_{\text {oc }}(\mu \mathbf{V})$ \\
\hline $\mathbf{A g}-\mathbf{N i}$ & 0.051 & 1.096 \\
\hline $\mathbf{A u}-\mathbf{N i}$ & 0.046 & 0.989 \\
\hline $\mathbf{C u}-\mathbf{N i}$ & 0.049 & 1.036 \\
\hline $\mathbf{T i}-\mathbf{N i}$ & 0.193 & 4.282 \\
\hline
\end{tabular}

\section{CONCLUSIONS}

Metallic nanoantennas can be tuned to absorb electromagnetic energy at infrared wavelengths. Through modifying geometries is possible have different broadband of energy harvested. We analyzed optical response of different metals getting the induced current on the nanoantennas, the results show that titanium at high frequencies has the best response. In thermal analysis we obtained the difference temperature along of the nanoantenas, the higher difference was for the junction Ti-Ni and its open circuit voltage was $4.282 \mu \mathrm{V}$. Large arrays of nanoantennas that act as thermocouples can be implemented to increase voltage response. Thermo-electrical nanoantennas can work together with traditional solar cells to increase the harvest of spectral energy.

\section{ACKNOWLEDGMENTS}

The author B. Mora Ventura acknowledges the scholarship of the Consejo Nacional de Ciencia y Tecnología

(CONACYT), also the Universidad de Guadalajara and the Universidad Autónoma de San Luis Potosí for the support to accomplish this research. FJG would like to acknowledge support by project 32 of "Centro Mexicano de Innovación en Energía Solar" from Fondo Sectorial CONACYT-Secretaría de Energía-Sustentabilidad Energética and by the National Laboratory program from CONACYT through the Terahertz Science and Technology National Lab (LANCYTT).

\section{REFERENCES}

[1] Garret M. and Sachit G., "Rectenna Solar Cells”, New York: Springer, pp. 231-257, (2013).

[2] Kotter D., Novack S., Slafer W., and Pinhero P., "Theory and manufacturing processes of Solar Nanoantenna Electromagnetic Collectors, ”J. Sol. Energy Eng., vol. 132(1), pp.011014(9 pages), (2010).

[3] Novotny L. and van Hulst N., “Antennas for light”, Nat. Photonics,vol. 5, pp. 83-90, (2011).

[4] Biagioni P, Huang JS, Hecht B., "Nanoantennas for visible and infrared radiation”, Reports on Progress in Physics 75.2: 024402, (2012).

[5] González F. J. and Boreman G., "Comparison of dipole, bowtie, spiral and log-periodic IR antennas," Infrared Phys. Technol. 46(5), 418-428, (2005).

[6] Hagerty J. A., Helmbrecht F. B., McCalpin W. H., Zane R., and Popovic Z. B., "Recycling ambient microwave energy with broad-band rectenna arrays," IEEE Trans. Microw. Theory Tech. 52(3), 1014-1024, (2004). 
[7] Bean J.A., Tiwari B., Bernstein G.H., Fay P. and Porod W., "Thermal infrared detection using dipole antennacoupled metal-oxide-metal diodes," J. Vac. Sci. Technol. B, vol. 27(1), pp. 11-14, (2009).

[8] Zhu Z., Joshi S., Grover S., and Moddel G., "Graphene Geometric Diodes for Terahertz Rectennas," J. Phys. D: Appl. Phys., vol. 46, pp. 185101 (2013).

[9] B. Edgar, Alda Javier and G.Francisco J., "Conversion efficiency of broad-band rectenas for solar energy harvesting applications", Optics Express, vol. 21, Issue S3, pp. A412-A418 (2013).

[10] Vandenbosch G. A. E. and Ma Z., "Upper bounds for the solar energy harvesting efficiency of nano-antennas," Nano Energy 1(3), 494-502 (2012).

[11] Ma Z. and Vandenbosch G. A. E., "Optimal solar energy harvesting efficiency of nano-rectenna systems," Sol. Energy 88, 163-174 (2013).

[12] Briones, E., et al, "Seebeck nanoantennas for the detection and characterization of infrared radiation," Optics express, 22(106), A1538-A1546 (2014).

[13] Graf A., Arndt M., Sauer M. and Gerlach G., "Review of micromachined thermopiles for infrared detection," Meas. Sci. Technol., vol. 18, pp. R59-R75, (2007).

[14] krenz P.M., Tiwari B.T., Szakmany G.P., Orlov A.O., Gonzalez F.G. and Boreman G.D., "Response Increase of IR Antenna-Coupled Thermocouple Using Impedance Matching," J. Quantum Electron., vol. 48(5), pp. 659-664, May (2012).

[15] Szakmany G.P., Krenz P.M., Orlov A.O., Bernstein G.H. and Porod W., "Antenna-Coupled Nanowire Thermocouples for Infrared Detection,” IEEE Trans. Nanotechnol., vol. 12(2), pp. 163-167, March (2013).

[16] Gonzalez F. J., Alda J., Simon J., Ginn J., and Boreman G., "The effect of metal dispersion on the resonance of antennas at infrared frequencies," Infrared Physics and Technology, vol. 52, no. 1, pp. 48-51, (2009).

[17] Palik, Edward D. Handbook of optical constants of solids. Vol. 3. Academic press, (1998).

[18] Ordal, Mark A., et al. "Optical properties of fourteen metals in the infrared and far infrared: $\mathrm{Al}, \mathrm{Co}, \mathrm{Cu}, \mathrm{Au}, \mathrm{Fe}, \mathrm{Pb}$, Mo, Ni, Pd, Pt, Ag, Ti, V, and W.” Applied optics 24.24: 4493-4499 (1985).

[19] Ordal, Mark A., et al. "Optical properties of $\mathrm{Au}, \mathrm{Ni}$, and $\mathrm{Pb}$ at submillimeter wavelengths." Applied Optics 26.4:744-752 (1987).

[20] Kovetz A., “The Principles of Electromagnetic Theory”, Cambridge University Press, Cambridge, UK (1990).

[21] Incropera F. P. and De Witt D. P., "Fundamentals of Heat and Mass Transfer", 4th ed., John Wiley \& Sons, New York (1996).

[22] Cuadrado, Alda, J., and González, F. J., "Multiphysics simulation for the optimization of optical nanoantennas working as distributed bolometers in the infrared", Journal of Nanophotonics, 7(1), 073093-073093, (2013).

[23] Ioffe, Abraham Fedorovich. "Semiconductor thermoelements and thermoelectric cooling." (1957).

[24] Rowe, David Michael, ed. “Thermoelectrics handbook: macro to nano”. CRC press, (2005).

[25] Graf A., Arndt M., Sauer M., and Gerlach G., "Review of micromachined thermopiles for infrared detection," Meas. Sci. Technol. 18(7), R59-R75 (2007).

Proc. of SPIE Vol. $956295620 \mathrm{P}-9$ 